Ann. Génét. Sél. anim., I976, 8 (4), 449-460.

\title{
MARQUEURS GÉNÉTIQUES SANGUINS CHEZ LES CHEVAUX DE TRAIT EN FRANCE
}

\author{
Marie KAMINSKI, A. VAN DE WEGHE *, Y. BOUQUET * et Luba PODLIACHOUK ** \\ avec la collaboration technique de Mmes Roselyne Beaud, Françoise Pigache, \\ de Mlle Michele Sykiotis et de M. J. M. Genna \\ Laboratoire d'Enzymologie du C.N.R.S., 91190 Gif-sur-Yvette, France \\ * Faculté de Médecine Vétérinaire, Université de Gand, \\ 9220 Merelbeke (Belgique) \\ ** Société d'Encouragement pour l'Amélioration des races de chevaux en France, \\ détachée au Laboratoire de Génétique Biochimique, \\ Centre national de Recherches zootechniques, $I$. N. R. A., \\ 78350 Jouy-en-Josas, France
}

\section{RÉSUMÉ}

Le polymorphisme génétique des constituants sanguins, exprimé par 7 systèmes d'antigènes érythrocytaires et par 8 systèmes de protéines dont 5 enzymes, a été analysé et comparé dans 6 races de chevaux de trait français : Ardennais, Boulonnais, Breton, Cob Normand, Comtois et Percheron.

Ces systèmes "marqueurs " définissent, pour chacune de ces races, un a profil génétique " caractéristique, qui, de plus, permet de distinguer les chevaux de trait des chevaux "de sang ", en particulier du Pur Sang Anglais.

La variabilité génétique des chevaux de trait est notablement plus marquée que celle des chevaux de sang; le nombre d'allèles est supérieur et les fréquences sont différentes pour les alleles communs aux deux groupes.

\section{INTRODUCTION}

L'étude comparée des marqueurs génétiques sanguins de chevaux, a montré, dès les résultats préliminaires (PoDLIACHOUK et KAMINSKI, I972), des différences notables entre le groupe des chevaux " de courses " et celui des chevaux de trait. Le premier groupe, comprenant des Pur Sang Anglais, Arabes, Trotteurs et Angloarabes, a été analysé récemment en détail, en comparant des populations issues de divers pays (PoDLIACHouk et al., I976).

L'intérêt majeur de l'étude des chevaux de trait est qu'ils dérivent pour la plupart des types équins locaux, connus depuis des temps très anciens, et à ce titre représentant un "capital génétique " transmis à travers des siècles. Ces populations 
ont été généralement produites dans une région bien délimitée et soumises à un type de sélection déterminé. Bien qu'il ait pu y avoir, au cours des âges, quelques apports extérieurs, il n'y a pas eu de mélanges entre ces groupes et ainsi les races résultantes ont une individualité bien marquée.

L'usage des chevaux en traction et en agriculture baisse actuellement rapidement et cette régression a entraîné une diminution très sensible des effectifs; leur utilité économique est justifiée à notre époque par la production de viande de boucherie.

\section{MATÉRIELS ET MÉTHODES}

\section{A. - Races (Quirter et Blanc, r974)}

I. Le cheval Ardennais, qui serait le descendant des chevaux de Solutré, présente un type façonné par le milieu environnant. L'élevage se fait actuellement dans l'indigénat, et on exporte souvent des étalons $A r d e n n a i s$ utilisés à rendre plus volumineux d'autres chevaux.

2. L'origine du cheval Boulonnais remonterait aux chevaux des légions de César, pour la plupart d'origine arabe, ayant fait souche en Bretagne. Des croisements fréquents avec les Arabes pratiqués au Moyen Age, ainsi que des croisements ultérieurs avec des lourds chevaux de Mecklembourg et des Genêts d'Espagne ont définitivement façonné la race, connue depuis le XVII ${ }^{\mathrm{e}}$ siècle.

3. Le cheval Breton a été formé dès le Moyen Age, par des croisements des juments locales avec des Arabes; plus tard, même des Pur Sang Anglais furent utilisés, et enfin des Hackneys. On distingue deux types, le Trait Breton et le Postier Breton; dans le présent travail ils ont été groupés.

4. Le Cob Normand est un demi-sang, sélectionné pour la traction. Il diffère du Cob Anglais qui est un cheval à deux usages. La jumenterie $C o b$ normande sert actuellement de base à l'élevage du cheval de selle en France.

5. Le cheval Comtois, introduit très anciennement par les Burgondes, est un cheval rustique, élevé actuellement dans l'indigénat.

6. Le cheval Percheron est marqué, comme le Boulonnais, par le sang Arabe, et ce depuis les Croisades. Le Percheron sélectionné est en fait un métis d'A rabe et de cheval indigène, présentant le type morphologique d'un Arabe grossi. La variété à robe noire est particulièrement prisée en Amérique.

\section{B. - Echantillons}

La plupart des échantillons ont été examinés entre 1972 et 1974, et proviennent des étalons des Haras nationaux, sauf pour le groupe des Boulonnais, constitué par des familles.

En tout, plus de 900 chevaux ont été analysés; pour des raisons techniques, tous les systèmes n'ont pas été déterminés sur tous les échantillons.

\section{C. - Techniques}

La détermination des antigènes érythrocytaires a été effectuée à l'aide de 26 antisérums spécifiques monovalents. 25 facteurs (antigènes) appartiennent à 7 systèmes génétiques distincts. Pour I6 d'entre eux la nomenclature internationale a été adoptée en 1974 et en 1976. Les techniques électrophorétiques et les procédés de révélation d'activités enzymatiques ont été décrits antérieurement (KaminsKi et al., 1974); des modifications ont été apportées depuis dans la détermination de transferrine (Nikolajczuk ex Balbierz, 1973) et d'estérase (BRAEND, 1970).

\section{RÉSULTATS}

\section{I. - Groupes sanguins}

Les fréquences des antigènes érythrocytaires sont données dans le tableau $r$. On peut constater que les Boulonnais diffèrent des autres par des fréquences les 
plus faibles des facteurs $A c, D c, F 8, F 14, P b$ et $U a$, et par des fréquences les plus fortes des facteurs $D a, Q a$ et $F$ 12. Les Comtois diffèrent de l'ensemble des autres races par des fréquences les plus faibles des facteurs $A a, D a$ et $J_{1}$ et la fréquence beaucoup plus forte que la moyenne du facteur $A c$. Enfin les Percherons se distinguent par la fréquence plus faible en $A c$ et, par contre, très élevée en $F 12$.

Dans le tableau 2 sont présentées les fréquences alléliques pour les systèmes simples, $C$ et $U$, et pour le système complexe $D$ (SANDBERG, I973; Podliachoux et al., I976). Ce dernier est constitué par au moins II allèles codominants : en effet, la découverte récente du facteur $G_{2}$, se comportant comme un sous-groupe du facteur précédemment dénommé $G$ (qui de ce fait devient $G_{1}$ ) change la composition du phénogroupe $D c e G 814$, qui devient : $D c e G_{1} G_{2} 814$. Un autre nouveau phénogroupe a, également, été trouvé : $D d G_{2}$.

Ainsi, dans l'état actuel de nos connaissances, le système génétique $\mathrm{D}$ comprend II antigènes, formant II phénogroupes, donc II allèles.

Pour le système $D$, un pourcentage important de génotypes peut être déduit directement des phénotypes: dans notre travail ce taux varie de $40 \mathrm{p}$. Ioo chez les Comtois à 96 p. Ioo chez les Boulonnais. De plus, il est possible de reconnaître un des deux allèles parmi les phénotypes restants, qui peuvent correspondre à plusieurs génotypes. Ainsi, le pourcentage des allèles identifiables devient 70 p. roo chez les Comtois et 98 p. xoo chez les Boulonnais.

Les fréquences alléliques du système $D$ ont été estimées par itération à partir des fréquences initiales des génotypes et des allèles reconnus. 4 à 5 itérations ont suffi à stabiliser les fréquences à la troisième décimale.

La comparaison des fréquences alléliques des groupes sanguins montre une analogie génétique entre les chevaux de trait, mis à part les Boulonnais.

Ainsi le phénogroupe (allèle) Aad est fréquent, sauf chez les Comtois qui, par contre, présentent $78 \%$ de phénogroupe Ace, relativement peu représenté dans les autres races de trait et inexistant chez les chevaux de sang. Les phénogroupes $A b d$ et $A b$ ont montré des fréquences moyennes; les échantillons manquant d'antigènes du système $A$ étaient rares.

L'allèle Ua est assez fréquent surtout chez les Ardennais; l'allèle $K a$, par contrf. présent chez les chevaux de sang, n'a pas été rencontré chez les chevaux de trait.

Dans le système $D$, les phénogroupes $D a d$ et $D d e$, absents chez le Pur Sang Anglais (Podliachouk et al., 1976) sont fréquents chez les chevaux de trait surtout Dad chez les Boulonnais. La fréquence du phénogroupe $D d 814$, faible chez les chevaux de course, est très élevée chez les chevaux de trait. D'autre part, ces derniers ne possèdent pas, ou très peu de phénogroupes $D d, D d f, D b c 14$ et $D c e G 814$, plutôt fréquents chez les Pur Sang Anglais ou les Trotteurs. Le phénogroupe Dcef 814 , trouvé jusqu'ici seulement chez les Trotteurs, n'a été détecté chez aucun cheval de trait. Enfin l'allèle Dc 8 I4, bien qu'absent chez les Boulonnais, se retrouve aussi bien chez les chevaux de trait que chez ceux de sang ou les Trotteurs.

\section{2. - Polymorphisme électrophorétique}

I es tableaux 3 et 4 donnent la répartition des phénotypes et l'estimation des fréquences géniques des divers systèmes dans les races étudiées. 


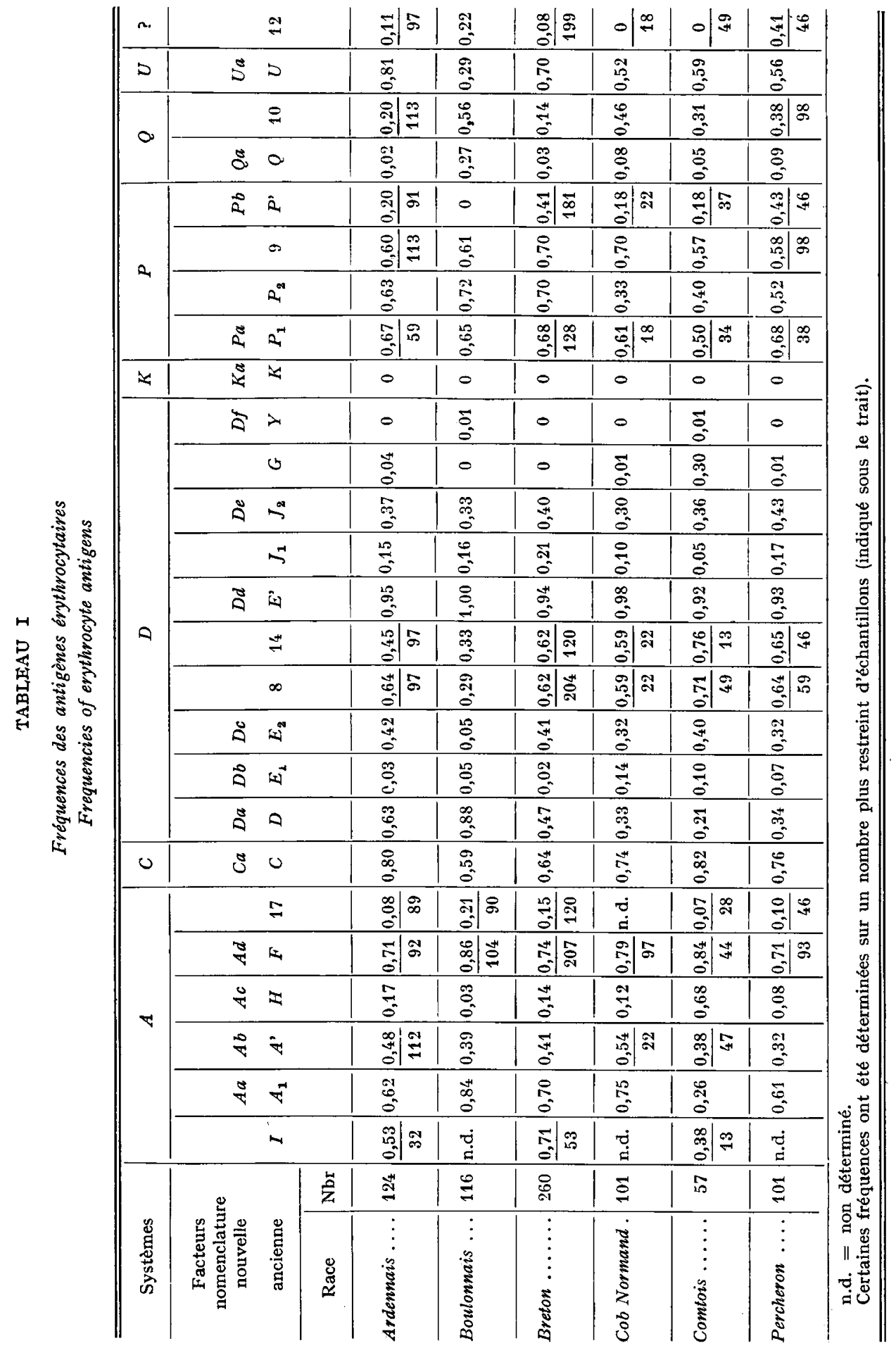


TABLEAU 2

A. Fréquences alléliques pour les systèmes $\mathrm{C}$ et $\mathrm{U}$ Allelic frequencies for the $\mathrm{C}$ and $\mathrm{U}$ systems

\begin{tabular}{|c|c|c|c|}
\hline & Nombre & $\mathrm{Ca}$ & Ua \\
\hline Ardennais $\ldots \ldots \ldots \ldots \ldots \ldots$. & 124 & 0,55 & 0,56 \\
\hline 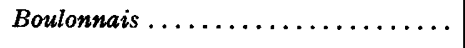 & 116 & 0,36 & 0,16 \\
\hline 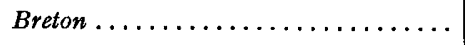 & 260 & 0,40 & 0,45 \\
\hline Comtois $\ldots \ldots \ldots \ldots \ldots \ldots \ldots$ & 457 & 0,58 & 0,36 \\
\hline Percheron ................... & 101 & 0,51 & 0,34 \\
\hline
\end{tabular}

$B$. Fréquences alléliques comparées pour le système $D$ chez les chevaux de trait, les Pur Sang Anglais et les Trotteurs

Allelic frequencies for the system $D$ in Draft, Thoroughbred and Trotters

\begin{tabular}{|c|c|c|c|c|c|c|c|}
\hline Phénogroupes & $\begin{array}{c}\text { Ardennais } \\
\mathrm{n}=90\end{array}$ & $\begin{array}{c}\text { Boulonnais } \\
\mathrm{n}=117\end{array}$ & $\begin{array}{l}\text { Bretons } \\
\mathrm{n}=200\end{array}$ & $\begin{array}{l}\text { Comtois } \\
\mathrm{n}=60\end{array}$ & $\begin{array}{l}\text { Percherons } \\
\text { n }=87\end{array}$ & $\begin{array}{c}\text { Pur Sang } \\
\text { Anglais } \\
\mathrm{n}=530\left(^{*}\right)\end{array}$ & $\begin{array}{l}\text { Trotteurs } \\
\mathrm{n}=1500\end{array}$ \\
\hline$D d \ldots \ldots \ldots \ldots \ldots$ & 0 & 0 & 0,090 & 0,188 & 0,108 & 0,156 & 0,144 \\
\hline$D a d \ldots \ldots \ldots$ & 0,367 & 0,615 & 0,278 & 0,106 & 0,204 & 0 & 0,074 \\
\hline$D d e . . . \ldots \ldots \ldots$ & 0,085 & 0,096 & 0,121 & 0,176 & 0,158 & 0 & 0,053 \\
\hline$D d f \ldots \ldots \ldots \ldots$ & 0 & 0 & 0 & 0 & 0 & 0,025 & 0,109 \\
\hline$D b c 14 \ldots \ldots \ldots$ & 0,011 & 0,030 & 0,013 & 0,017 & 0,029 & $0,19 \dot{x}$ & 0,075 \\
\hline$D c 814 \ldots \ldots \ldots \ldots$ & 0,188 & 0 & 0,220 & 0,133 & 0,144 & 0,182 & 0,295 \\
\hline$D d e J_{1} \ldots \ldots \ldots \ldots$ & 0,089 & 0,093 & 0,106 & 0,017 & 0,097 & 0,068 & 0,173 \\
\hline$D d 814 \ldots \ldots \ldots \ldots$ & 0,237 & 0,167 & 0,173 & 0,348 & 0,254 & 0,025 & 0,070 \\
\hline Dcef $814 \ldots \ldots \ldots$ & 0 & 0 & 0 & 0 & 0 & 0 & 0,006 \\
\hline Dce $G 814 \ldots \ldots \ldots$ & 0,023 & 0 & 0 & 0,017 & 0,006 & 0,142 & 0,003 \\
\hline
\end{tabular}

$\left(^{*}\right)$ Il restait $2 \mathrm{I}$ p. Ioo de phénotypes non interprétés.

Pour 1'Albumine les fréquences de $A 1^{F}$ sont toujours plus faibles que celles de $A 1^{S}$; les variations entre les races sont peu importantes.

Dans le système de Transferrine, 1'allèle $T f^{F}$ prédomine et sa fréquence moyenne est très voisine de celle du Pur Sang Anglais : 0,457 contre 0,474 . Pour $T f^{D}$ les écarts entre les populations sont très sensibles et il est, en moyenne, moins fréquent chez les chevaux de trait que chez les chevaux de sang: 0,I38 contre 0,315. $T f^{H}$, le plus fréquent chez les Ardennais, est en moyenne deux fois plus fréquent chez les chevaux de trait que chez ceux du sang. Enfin $T f^{R}$, rare chez les chevaux de course et totalement absent chez les Arabes d'origine diverse, est relativement fréquent chez les chevaux de trait : moyenne 0,137 .

Sur le plan de phénotypes, les effectifs attendus diffèrent d'une façon significative des observés pour les Ardennais, Bretons et Comtois. 


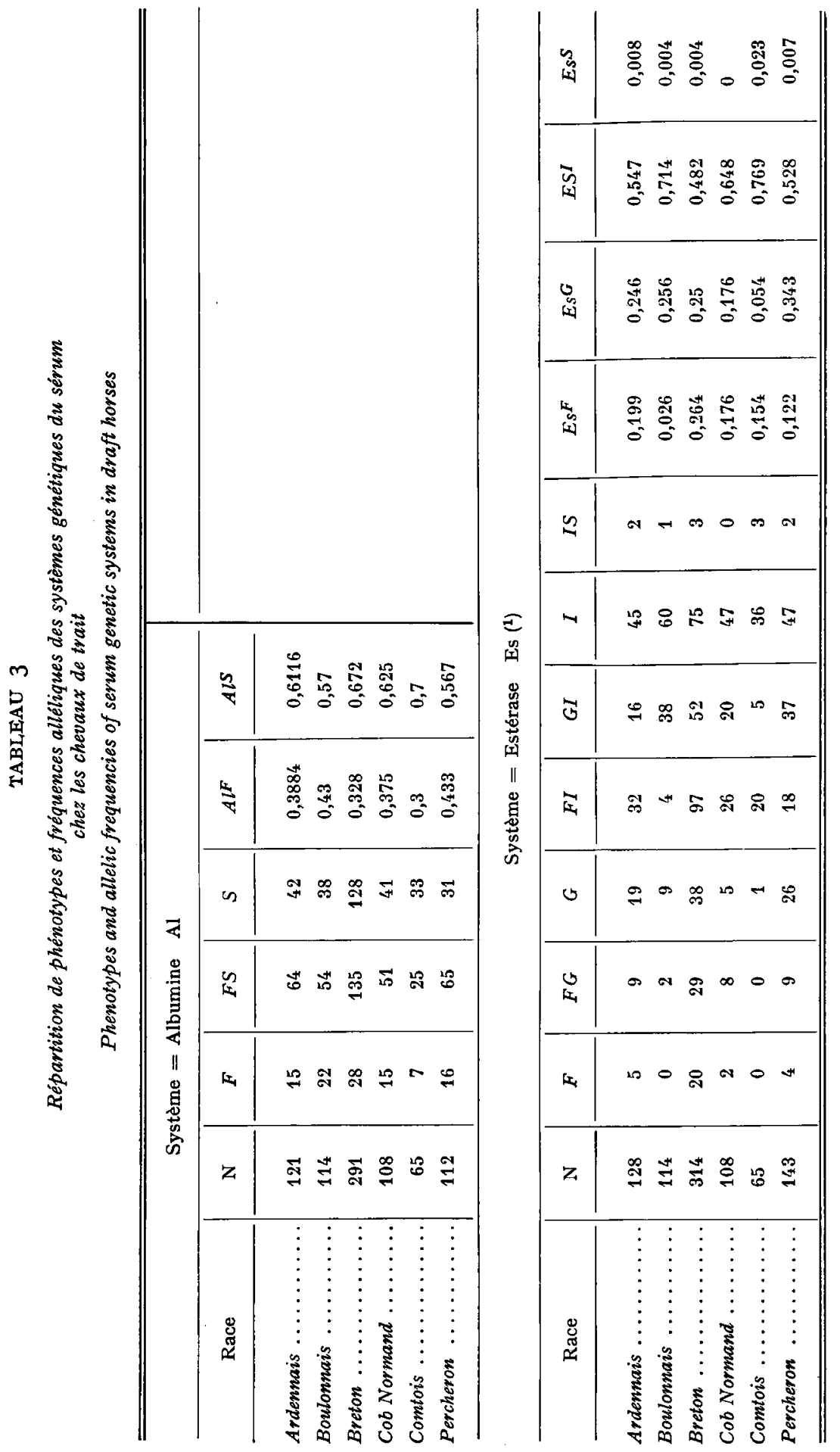




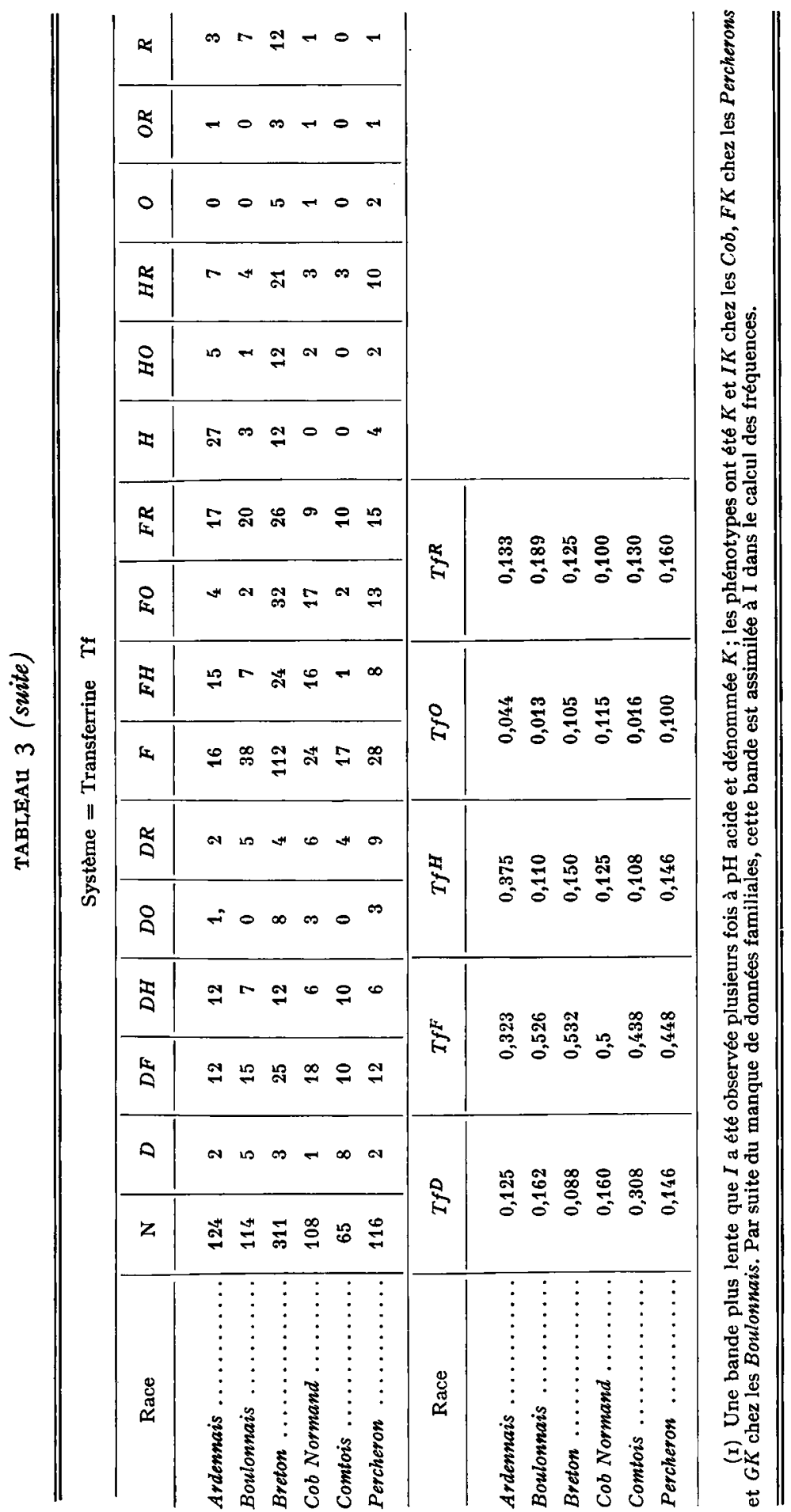



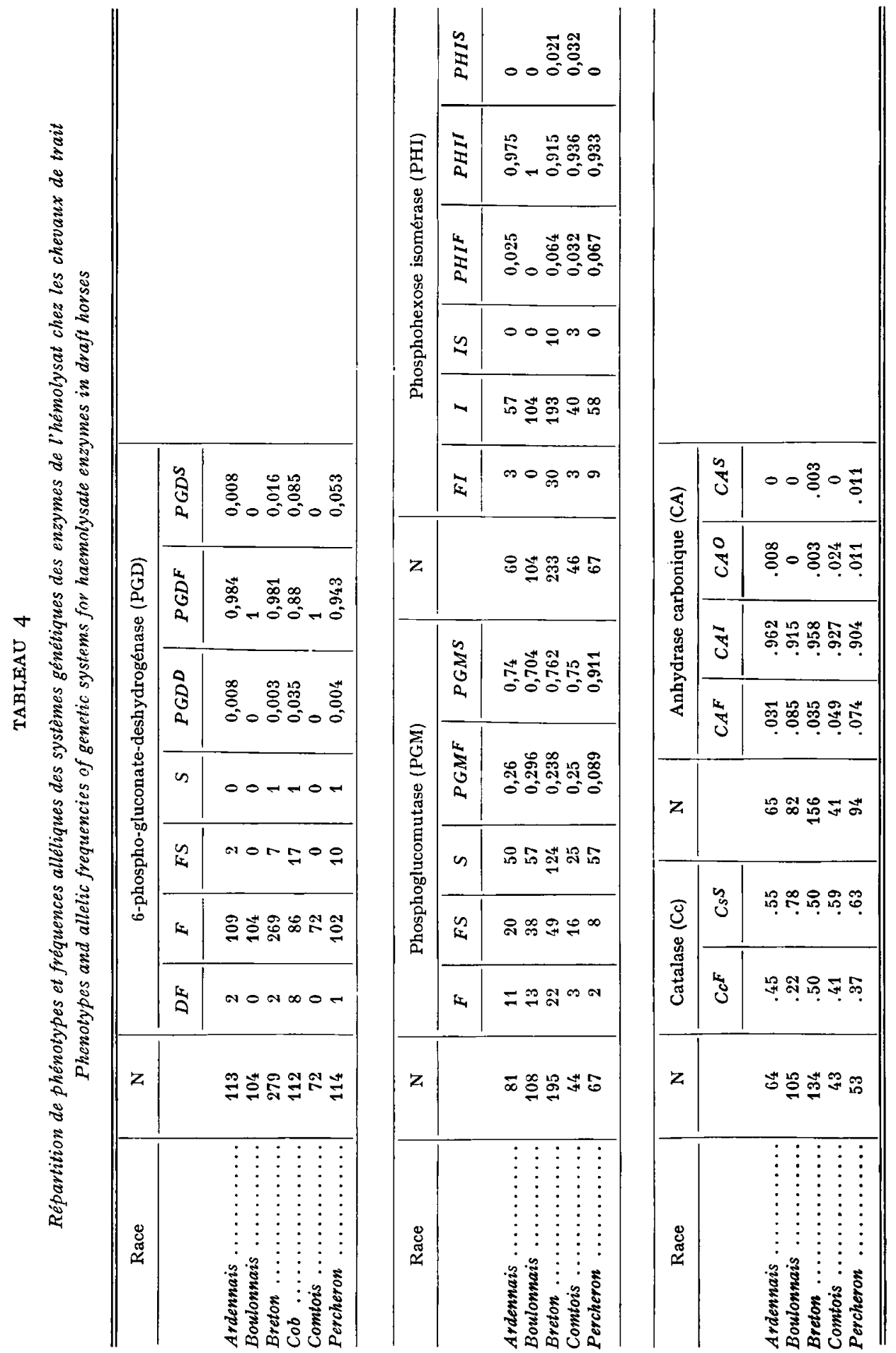
Pour l'Estérase, la caractéristique la plus frappante des chevaux de trait est la fréquence relativement élevée de $E S^{G}$ (0,238 en moyenne) alors que ce variant est absent chez les Pur Sang Anglais et que sa fréquence chez les chevaux de demisang est 0,047 pour les Trotteurs et 0,065 pour le Selle Français.

Parmi les populations étudiées, les fréquences de $E s^{G}$ et de $E s^{F}$ varient sensiblement; la fréquence moyenne de $E s^{F}$ est de 0,183 , alors que chez les chevaux de course elle n'est que de 0,028 . L'allèle_Es $s^{I}$ par contre est bien moins fréquent chez les chevaux de trait, surtout les Bretons.

Les effectifs des phénotypes $G$ et $G I$ observés diffèrent de façon significative des effectifs attendus chez les Ardennais, les Bretons et les Percherons.

Parmi les enzymes de l'hémolysat, le système de la 6-phosphogluconate déshydrogénase présente une différence marquée entre le groupe des chevaux de trait et celui des chevaux de course. Chez les premiers on a trouvé la bande $D$, absente chez les seconds. De plus, la fréquence moyenne de $P D G^{F}$ est de 0,97 chez les trait, alors qu'elle est de $0,58 \mathrm{chez}$ les Pur Sang Anglais. Ainsi le phénotype $S$ n'a été trouvé que chez seulement 3 chevaux de trait sur 775 examinés contre I43 sur 843 Pur Sang Anglais.

Pour la Phosphoglucomutase, 1'allèle le plus fréquent est $P G M^{S}$. Chez les chevaux de trait on en trouve en moyenne $89 \%$ contre roo \% chez les Pur Sang Anglais et $94 \%$ chez les Trotteurs. La présence de $P G M^{F}$ chez les chevaux de trait se manifeste par la fréquence appréciable d'individus de phénotype $F$ : on en a trouvé ro \% contre I \% chez les Selle Français et les Trotteurs.

Dans le système de la Phosphohexose-isomérase on note la présence de $P H I^{S}$ chez les Bretons et les Comtois; cet allèle n'a pas été trouvé chez les chevaux de course. La fréquence de l'allèle prépondérant $\left(P H I^{I}\right)$ est la même chez les chevaux de trait et de course : 0,94 et 0,97 en moyenne.

Parmi les allèles rares, connus à ce jour chez le Cheval, nous n'avons pas rencontré chez les chevaux de trait français de $A l^{I}, T f^{M}$ ni $P G M^{V}$. Remarquons que ce dernier est présent chez les chevaux français de demi-sang.

La Catalase, presque monomorphe chez les Pur Sang Anglais, présente chez les chevaux de trait des fréquences avoisinant 0.5 pour chacun des allèles. Quant à l'Anhydrase carbonique, malgré la prépondérance de $1^{\prime}$ allèle $\mathrm{CA}^{I}$, on trouve chez les chevaux de trait les variants $C A^{S}$ et $C A^{O}$, absents chez le Pur Sang Anglais (tabl. 5).

Une estimation de la variabilité génétique par l'utilisation de II systèmes marqueurs sanguins a été effectuée en multipliant les valeurs $\left(\Sigma p^{4}+\Sigma 4 p^{2} q^{2}\right)$ obtenues pour chaque système individuel. Ces estimations nous renseignent sur le nombre d'individus dans un million pouvant posséder le même génotype pour tous les systèmes envisagés : Ardennais I9, Boulonnais I9o, Breton II, Comtois 294 et Percheron Io. La variabilité génétique pour les systèmes considérés est donc nettement moindre chez le Boulonnais et Comtois que dans les autres populations.

Une mesure de distance génétique moyenne fut appliquée afin de se rendre compte de la différence génétique séparant 5 populations de chevaux de trait (SoKAL, and SNEATH, I963). Le paramètre $D_{k}$ dénote la distance génétique entre les populations $a$ et $b$ pour le locus $k$. Si $X_{i a}$ et $X_{i b}$ représentent les fréquences de l'i-ème 
allèle dans les populations $a, b$, et n'étant le nombre d'allèles pour le locus $k$, la valeur de $D_{k}$ est : $\quad D_{k}=\left(\sum_{i=I}^{n}\left(X_{i a}-X_{i b}\right)^{2}\right)^{\frac{1}{2}}$

La valeur de $\mathrm{D}$ peut fluctuer entre o et $\mathrm{I,4}$ I par locus. La globalisation simultanée des distances génétiques fournies par plusieurs loci s'exprime par le coefficient $\mathbf{D}$ moyen $=\frac{\mathbf{r}}{\mathbf{x}} \sum_{\mathbf{k}=I}^{\mathbf{X}} \mathrm{D}_{\mathbf{k}}$, $\mathrm{x}$ étant le nombre de loci pris en considération.

Les coefficients de distances génétiques moyens pour II systèmes de marqueurs sanguins sont donnés dans le tableau 6 pour 5 populations de chevaux de trait. On voit par exemple que l'Ardennais diffère davantage du Boulonnais que le Breton du Percheron.

TABLEAU 5

Distances génétiques moyennes entre 5 populations de chevaux de trait Mean genetic distances between five draft horses populations

\begin{tabular}{l|c|c|c|c|c}
\hline \hline \multicolumn{1}{c|}{ Race } & 1 & 2 & 3 & 4 & 5 \\
\cline { 2 - 4 } & & & & \\
1. Ardennais....... & 0 & .2645 & .1643 & .1450 & .1455 \\
2. Boulonnais ...... & & 0 & .1977 & .2042 & .1311 \\
3. Breton ......... & & & 0 & .1436 & .1289 \\
4. Comtois ......... & & & & 0 & .1354 \\
5. Percheron ....... & & & & & 0 \\
\hline \hline
\end{tabular}

\section{DISCUSSION ET CONCLUSIONS}

Des études portant sur un nombre limité de marqueurs génétiques sanguins ont été publiées, concernant quelques races et populations. A côté de résultats similaires (PoDliachouk et al., I962; PoDliachouk et al., I966), la comparaison avec nos données présentes montre quelques divergences.

Ainsi, chez les 6r Ardennais de Suède (BEngtsson et al., I968) on a trouvé moins de $T f^{D}$ et $T f^{H}$ et davantage de $T f^{O}$ et $T f^{R}$. Pour l'estérase, seule la séparation à $\mathrm{pH}$ alcalin a été effectuée de sorte que la fréquence rapportée de $E s^{F}$ correspond en fait à la somme de $E s^{F}+E s^{G}$ et dans ces conditions les résultats suédois sont similaires aux nôtres. De même, les 5I Percherons d'Afrique du Sud (Osterfhoff and WARD-Cox, I967) montrent une fréquence de $E s^{F}$ compatible avec nos résultats. Par contre, les résultats diffèrent sensiblement pour l'Albumine; $A l^{F} 0,67$ et $A l^{S}$ 0,33 , et pour la Transferrine : davantage de $T f^{F},(0,7)$ et nettement moins de $T f^{H}$ $(0,05)$ et $T f^{R}(0)$. De telles divergences peuvent être imputées soit à 1'échantillonnage non représentatif de l'ensemble de la population soit aux réelles différences de souche.

En général, nos résultats démontrent que certains systèmes génétiques présentent un même degré de variabilité chez les chevaux de tous les types - ce sont l'albumine et la transferrine. Par contre, une population peut présenter une variabilité génétique 
marquée dans un système et le monomorphisme dans un autre, alors que l'on observe l'inverse dans une autre race. Ainsi, les Pur Sang Anglais présentent un polymorphisme accentué pour le 6-PDG et sont très peu polymorphes pour l'estérase, alors que chez les chevaux de trait le polymorphisme de l'estérase est bien développé et celui de 6-PGD l'est peu.

En relation avec l'origine et la différentiation des races des chevaux de trait françaises, il était intéressant de rechercher dans quelle mesure l'ascendance arabe des Boulonnais et des Percherons était perceptible à travers leur " profil génétique sanguin " actuel. Ces deux races présentent une faible fréquence de l'antigène Ac, absent chez les Arabes et plutôt fréquent chez les autres chevaux de trait. Par contre, l'antigène $\mathrm{Da}$, absent chez les Arabes, se trouve chez les Boulonnais au taux le plus élevé parmi les chevaux de trait. De même l'allèle $T f^{R}$, non trouvé chez les Arabes, présente chez les Boulonnais et chez les Percherons des fréquences les plus fortes parmi tous les chevaux de trait.

On ne peut donc, sur la base des données génétiques sanguines actuelles, retrouver la lointaine ascendance Arabe chez les Boulonnais ni chez les Percherons. Les pressions du milieu environnant et celles de la sélection sont allées à l'encontre du patrimoine génétique originel, en favorisant l'apport d'autres variants et leur maintien dans ces deux races.

En conclusion, 1'ensemble de résultats portant sur plusieurs systèmes génétiques permet de distinguer 3 catégories de chevaux qui correspondent aux types fonctionnels : les chevaux de sang (Pur Sang Anglais, Arabe, Anglo-Arabe) contrastant à l'extrême avec les chevaux de trait, tandis que les chevaux de demi-sang (Trotteur, Selle Français) occupent une position intermédiaire. Une telle différenciation catégorielle est basée sur l'absence de certains allèles tels les phénogroupes $D d f, D c e f 8 \mathrm{r} 4$, $K a$ chez les chevaux de trait, ou les phénogroupes $A c e, D a d$, et allèles $6-P G D^{D}$, $P G M^{F}, P H I^{F}, P H I^{S}, C A^{o}, E s^{G}$ chez le Pur Sang Anglais, ainsi que des différences marquées dans les fréquences alléliques, surtout pour 6-PGD, Es, Tf.

En général, dans la majorité des systèmes la variabilité est plus accusée chez les chevaux de trait que chez les chevaux de sang, ce qui se traduit par un taux élevé de l'efficacité dans les contrôles de filiation et d'identification.

Reçu pour publication en septembre 1976.

\section{REMERCIEMENTS}

Ce travail a bénéficié du support financier de la Société d'Encouragement pour l'Amélioration des races de chevaux en France (Directeur J. RoMANET), ainsi que de l'Institut pour l'Encouragement de la Recherche Scientifique dans l'Industrie et l'A griculture (Bruxelles).

Les auteurs remercient vivement M. Blanc, le Directeur du Service des Haras, d'avoir mis à notre disposition les échantillons de sang des étalons des Haras nationaux, ainsi que M. CALAIs, Président de la Fédération du Cheval Boulonnais, et le Dr. vét. D. Desquines, qui ont mis à notre disposition les échantillons de sang des chevaux Boulonnais.

Les auteurs expriment leurs remerciements à Mmes R. Beaud, F. Pigache, Mlle M. Sykiotis et M. J. M. GenNa pour l'excellente collaboration technique. 


\section{SUMMARY}

\section{BLOOD GENETIC MARKERS IN FRENCH DRAUGHT HORSES}

The 6 populations studied were: I39 Ardennais, II 4 Boulonnais, 320 Bretons, II 4 Cob Normand, 74 Comtois, $15 \mathrm{I}$ Percherons.

The allelic frequencies of 7 systems of erythrocyte antigens and of 8 systems of polymorphic proteins of serum and haemolysate were determined.

The presence of certain alleles in $A, D$ and $K$ systems of erythrocyte antigens and in Esterase, 6-PGD, $P G M$ and $P H I$ systems, as well as notable differences between the allelic frequencies in the $D$ erythrocyte antigen system and $6-P G D, T f, E s$, allow to propose a classification of the different types of horses. The Thoroughbreds are markedly different from the draught horse populations, while Trotters occupy an intermediate position. The genetic variability is more pronounced in draught horses than in light horses.

\section{RÉFÉRENCES BIBLIOGRAPHIQUES}

Braend M., r97o. Genetics of Horse acidic Prealbumins. Genetics, 65, 495-503.

Bengtsson S., Gahne B., Rendel J., 1968. Genetic Studies on Transferrins, Albumins, Prealbumins and Esterases in Swedish Horses. Acta Agr. Scand., 18, 60-64.

Kaminski M., Bouguet Y., Van De Weghe A., Podliachouk L., 1974. Ontogenèse des marqueurs génétiques sanguins chez le Cheval. Ann. Génét. Sél. Anim., 6, 195-2ro.

Nikolajczuk M., Balbierz H., r973. Simultaneous Determination of Transferrin and Albumin Phenotypes in Horses. Arch. Immun. Ther. Exper. (Pol.), 21, 577-581.

Osterhoff D. R., WARD-Cox F. S., 1967. A preliminary Horse Breed Comparison with Regard to Haemoglobin and Serum Type Polymorphism. Proc. S. Afr. Soc. Anim. Prod., 218-223.

Podliachouk L., Kaczmarek A., Zwolinski J., i962. Les groupes sanguins des chevaux de six races de Pologne. Ann. Inst. Pasteur, 108, 943-949.

Podliachouk L., Kaminski M., I972. Studies on Blood Groups, Esterases and Transferrins in light and draught Horses. Abstr. X'IIIth Europ. Conf. Anim. Blood Grps Biochem. Polymorph. (Vienne). Anim. Blood Grps Biochem. Genet., 3, suppl. I, 46-47.

Podliachouk L., Kaminski M., VAN De Weghe A., Bouquet Y., Zwolinski J., Siudzinski S., 1976 Marqueurs Génétiques sanguins chez les chevaux de course. Ann. Génét. Sél. Anim., 7, 339-355.

Podliachouk L., Kaminski M., Zwolinski J., 1975. Étude des marqueurs génétiques sanguins dans deux races de Poneys de Pologne. Ann. Génét. Sél. Anim., \%, 167-180.

Ponliachouk L., Madeyska A., Desguines D., I966. Étude immunogénétique du cheval Boulonnais. Proc. Xth Conf. Eur. Soc. Blood Grps Biochem. Polymorph. Paris, 333-338.

Quittet E., Blanc H., 1974. Races chevalines en France. La Maison rustique, Paris 6.

SANDBERG K. I973. The D blood group system of the horse. Anim. Blood Grps. Biochem. Genet. 4 r93-205.

Sokal R. R. and SNeath P. H. A. (1963). Principle of numerical taxonomy, Freeman, San-FranciscoLondon. 